\title{
Fuentes para el estudio del movimiento obrero: El Servicio de Documentación e Información Laboral (DIL), dirigido por Leonardo Dimase (1960-1976, 1982-1989)
}

Sources for the study of the labor movement: The Documentation Service and Job Information (DIL), directed by Leonardo Dimase (1960-1976, 1982-1989)

\section{Darío Dawyd y Silvia Nassif}

\section{OpenEdition} Journals

\section{Edición electrónica}

URL: http://journals.openedition.org/corpusarchivos/604

DOI: 10.4000/corpusarchivos.604

ISSN: 1853-8037

\section{Editor}

Diego Escolar

\section{Referencia electrónica}

Darío Dawyd y Silvia Nassif, « Fuentes para el estudio del movimiento obrero: El Servicio de Documentación e Información Laboral (DIL), dirigido por Leonardo Dimase (1960-1976, 1982-1989)», Corpus [En línea], Vol 3, No 2 | 2013, Publicado el 20 diciembre 2013, consultado el 01 mayo 2019. URL : http://journals.openedition.org/corpusarchivos/604; DOI : 10.4000/corpusarchivos.604 


\section{Fuentes para el estudio del movimiento obrero: El Servicio de Documentación e Información Laboral (DIL), dirigido por Leonardo Dimase (1960-1976, 1982-1989)}

Sources for the study of the labor movement: The Documentation Service and Job Information (DIL), directed by Leonardo Dimase (1960-1976, 1982-1989)

Darío Dawyd y Silvia Nassif

\section{NOTA DEL EDITOR}

Fecha de recepción del original: 02/11/2013

Fecha de aceptación para publicación: 27/11/2013

\section{Introducción}

1 En la actualidad, los estudios sobre el movimiento obrero argentino han vuelto a tener un peso considerable. Los investigadores de este tema nos encontramos frecuentemente con la problemática de que las fuentes disponibles para su estudio -sobre todo para las décadas de 1960 y 1970, coincidentes con las dictaduras militares- resultan escasas y se encuentran de manera fragmentada. Los sindicatos generalmente no conservan archivos, otros fueron saqueados o su acceso es restringido, de manera que salvo excepciones, las publicaciones producidas por aquellos no suelen encontrarse en otros repositorios. ${ }^{1}$ De esta manera, la prensa informativa, como diarios y revistas, se constituye en una de las fuentes privilegiadas. ${ }^{2}$ También los archivos de las fuerzas de seguridad, recientemente

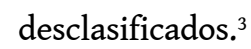


2 Al mismo tiempo, los Informes Laborales del Servicio de Documentación e Información Laboral (DIL) son también una fuente importante para el conocimiento del movimiento obrero, tanto de la Capital Federal y del Gran Buenos Aires, como de gremios y federaciones de distintas regiones del país. Fundado en 1960 y editado ininterrumpidamente hasta 1976, y vuelto a editarse entre 1982 y 1989, el DIL publicaba mensualmente los Informes Laborales, que recopilaban informaciones de diversos temas del mundo laboral (conflictos, convenios, movimientos en los nucleamientos sindicales, legislación, etc). Posteriormente editaron los Informes Políticos y otras publicaciones de diversa periodicidad, todas relacionadas con el mundo político y laboral. ${ }^{4}$

3 A lo largo de diversas investigaciones (Dawyd, 2011; Nassif, 2012) dimos con la utilización de los Informes Laborales como fuentes en diversos trabajos, aunque generalmente sin conocer quién los producía; otros investigadores no conocían la posibilidad de acceso a los mismos por la falta de información acerca de los lugares donde pueden consultarse, y otros no saben de su existencia. En solo tres diversos trabajos pudimos encontrar referencias a nuestro objeto:

"El informe DIL, una empresa de información sindical, cuyo boletín está destinado a las cúpulas sindicales y empresarias". (Duval, 1974, p. 302.)

"El Centro de Documentación e Información Laboral, fundado por Leonardo Dimasse, fue durante largos períodos una referencia casi única, precisa, sobre conflictos laborales, a través de su Boletín DIL. Fallecido su fundador, su hijo conservó aquel valioso patrimonio en la Fundación que lleva el nombre del autor, pero ignoramos si prosigue en la actualidad." (Izaguirre y Aristizábal, 2002, p. 16)

"Such data can be culled, however, from the monthly newsletter of the Servicio de Documentación e Informacion Laboral (DIL) in Buenos Aires. Founded in 1961 by the late Leonardo Dimase, DIL published from 1961 to 1976 an invaluable monthly newsletter called Informes Laborales, which included a section describing individually all the labor conflicts its researchers found out about each month. DIL was forced to close in the repressive atmosphere of the post-1976 military regime, but reopened in 1982 when the military announced its return to the barracks. From that point until June 1989, when the economic crisis forced DIL to close for lack of funds, the newsletter continued to appear. Although Informes Laborales lacked a systematic format for presenting its accounts of strikes (apart from grouping them by industry), and although the newsletter gave no systematic information on strike duration or number of participants, it provided key information on the stated causes of strikes that is not available elsewhere. The primary sources for the strike information in Informes Laborales were the major Buenos Aires newspapers and the DIL staffers' union and employer contacts around the country (interviews with DIL staffers, April 1985, September 1986, and June 1989). Of 2503 strikes recorded in Tendencias Economicas between January 1984 and May 1989, Informes Laborales reported the stated causes of 1,468." (McGuire, 1992, p. 6).

4 Existen distintas confusiones acerca de quiénes fueron sus hacedores y es posible pensar que, justamente, al no estar clara la procedencia de estos documentos, hasta el momento no fueron muy utilizados por los investigadores. Por este motivo, entendemos que este trabajo podría ayudar al conocimiento de este acervo documental para constituirse en una posible fuente para el estudio del movimiento obrero argentino. Los objetivos de este trabajo consisten en analizar los Informes Laborales del DIL, quiénes los elaboraron, a quiénes estaban dirigidos, de dónde recogían la información, cómo se financiaban, entre otras cuestiones. En segundo lugar, indicar en qué lugares físicos se encuentran disponibles en la actualidad para su consulta. Finalmente realizar una breve reseña de su fundador y director, Leonardo E. Dimase. 


\section{Documentación e Información Laboral}

5 El servicio de Documentación e Información Laboral presentó su primer Informe Laboral el 10 de marzo de 1960, bajo la dirección de Leonardo Enrique Dimase. ${ }^{5}$ Los temas del primer número fueron: conflictos, convenios, agrupamientos sindicales, vida sindical y legislación. En la página 2 incluía la presentación del nuevo emprendimiento:

"Servicios. La serie Informes, cuyo primer número ofrecemos hoy, no es sino una de las muchas manifestaciones de la tarea que DIL se ha propuesto desarrollar. A través de entregas sucesivas se tendrá, mes a mes, una visión amplia y objetiva de todo cuanto ocurra en el plano social y, en particular, en el campo laboral. Desde un panorama general informativo del acontecer diario, hasta la referencia legal en la materia, desde la aportación del mayor número de elementos de juicio acerca de todos y cada uno de los problemas - nuestra norma de absoluta objetividad dejará para cada uno las conclusiones pertinentes- hasta la noticia bibliográfica y la cita del antecedente, nacional o extranjero, sin descuidar por cierto la dilatada literatura acerca de las modernas técnicas de la organización científica del trabajo, nada será ajeno a las páginas de estos informes mensuales que esperamos ayuden a cubrir una necesidad sentida en la materia. Dijimos ya que esta Serie forma parte de un Plan de Trabajo mucho más vasto. En efecto, en nuestra Organización están dadas todas las condiciones para convertir a la misma en un verdadero centro documental e informativo especializado al cual puedan recurrir todos aquellos que, por razones de la actividad a que están dedicados o por simple inquietud, necesiten de una información o de un documento vinculado con los problemas objeto de nuestra dedicación. A manera de anticipo, y sin que ello implique una enumeración exhaustiva ni mucho menos excluyente, anotamos seguidamente algunos de los Servicios que, además del que constituye la serie Informes, estamos ya en condiciones de brindar."6

6 Entre aquellos servicios incluían la copia de documentación especializada, el análisis del proceso social argentino, referencias documentales y asesoramiento general.

7 Respecto a quiénes realizaban estos documentos, según relata Miguel Dimase, no había un plantel fijo, aunque había distintas personas que trabajaban con ellos, entre los que destaca a Horacio Eichelbaum, quien se dedicaba a la parte referida a los sucesos internacionales. También trabajó en el emprendimiento el ahijado de Dimase, Héctor Folgueira. Al mismo tiempo, Dimase destaca que su familia siempre cumplió un rol muy importante en la logística y sostenimiento de los DIL.

8 El rol del imprentero Pagani fue de suma importancia, ya que fue él quien ayudó en la inversión del capital inicial, y también en otros momentos ayudó a pagar algunas de las deudas que fueron contrayendo.

9 Con relación a cómo se recolectaba la información, Miguel Dimase explica que la misma era elaborada a partir de los distintos materiales que les proporcionaban los sindicatos (memorias, balances), notas de prensa, información de los diarios, solicitadas. Asimismo, también les brindaban información las agrupaciones sindicales. Todos los materiales recibidos eran recopilados y archivados, para brindar el servicio de documentación que ofrecía el DIL.

10 Los Informes Laborales tuvieron una tirada de entre 500 a 1000 ejemplares. La cantidad de ejemplares estaba íntimamente ligada a la cantidad de suscriptores, ya que esta era la principal fuente de financiamiento. En ese mismo sentido, afirmaban que el emprendimiento era “...financiado exclusivamente con el producido de las tarifas de 
suscripciones y asesoramientos". ${ }^{7}$ Cabe notar que, por ejemplo, para el año 1972 la suscripción anual de la serie Informes Laborales costaba 110 pesos de la época, es decir, menos de 10 pesos por mes, cuando las revistas de interés general como Primera Plana, Panorama y Análisis en el mismo año costaban 3 pesos por semana, unos 12 pesos al mes.

El primer espacio físico en el que funcionó el DIL fue en la propia casa de Leonardo Dimase, en la calle Teodoro Viladerbo 1815, a 15 cuadras de la Unión Vecinal de Villa del Parque, donde Dimase dictaba clases particulares y cursos. ${ }^{8}$ La primera máquina de escribir fue facilitada por Antonio A'gulo, canillita y referente de la zona. ${ }^{9}$

Con el correr de los años, a la Serie Informes Laborales añadieron en 1964 la Serie Informes Políticos ("Aparece mensualmente desde 1964. Exhaustivo análisis del proceso nacional”) y en 1972 la Serie Textos Legislativos (“Aparece mensualmente desde 1972. Contiene textos completos de leyes, decretos, resoluciones y proyectos legislativos, referidos a los campos laboral y previsional"). Al margen de estas tres publicaciones mensuales, con diversa periodicidad aparecieron la Guía de Organizaciones Gremiales ("Registro de más de 4000 organizaciones con domicilio, número telefónico y nómina de dirigentes"), Nucleamientos Sindicales ("Antecedentes y evolución de los núcleos que actúan en el campo sindical, con interesantes datos estadísticos"), y Remuneraciones ("Salario vital, mínimo y móvil. Antecedentes, legislación, escalas salariales por zonas, etc., incluidos cuadros sobre la evolución de las asignaciones familiares"). ${ }^{10}$ por compra directa. También había informes especiales, que aparecían generalmente al cumplirse algún aniversario de la fundación (como en febrero de 1970, cuando el DIL cumplió 10 años e hicieron en el Informe Especial № 111 un resumen del período posterior a 1955).

Desde el Informe Laboral № 1, de marzo de 1960, las secciones internas eran: conflictos en diferentes áreas laborales, convenios, agrupaciones sindicales, vida sindical (elecciones) y congresos; en los números siguientes fueron incorporando relaciones intersindicales, noticias en relación con resoluciones oficiales, legislación, noticias varias, resúmenes anuales, informes especiales (seguridad social, por ejemplo) y cursos que se dictaban en los sindicatos $u$ otras asociaciones. También algunos números incluían suplementos sobre diferentes temas; el primero de ellos fue el suplemento del Informe № 2 sobre comisiones mixtas entre funcionarios y sindicatos del transporte. Posteriormente también se incluyeron separatas sobre tendencias sindicales y la CGT.

En el informe № 103, de septiembre de 1968, agregaron una nueva sección denominada "interior", ya que "todo indica que los acontecimientos a nivel sindical más allá de la Capital Federal darán, en un futuro no lejano, pautas que incidirán en el orden nacional", según los editores debido a la aparición de conflictos como el del sindicato de Construcción de Córdoba y Tucumán, que rechazó la intervención del sindicato nacional, porque los primeros se habían adherido a la CGT de los Argentinos.

En el Informe Laboral № 159, de mayo de 1973, presentaron una nueva estructura interna del boletín, sin divisiones en conflictos, vida sindical, y las otras secciones, sino por actividad; así, todos los temas de cada actividad laboral se informaban alfabéticamente en cada una de ellas (elecciones, congresos, conflictos, convenios).

17 El Departamento de Consultoría y Asesoramiento Integral brindaba servicios que abarcaban desde consultas jurídicas hasta relativas a las obras sociales. El Departamento de Relaciones, daba asesoramiento en relaciones públicas y prensa (desde redacción hasta 
impresiones, desde preparación de congresos hasta servicio de filmación de los mismos). Finalmente, el Departamento de Capacitación daba cursos en materia laboral y previsional, así como en asesoramiento bibliográfico, prensa, relaciones públicas y difusión.

Desde el primer número hasta el de abril de 1967 el formato era de hoja tamaño A3 doblada a la mitad, en forma de libro. A partir de mayo de 1967 el formato pasó a tamaño carta, perforado.

Con relación a quiénes eran los suscriptores del DIL, Miguel Dimase señala que, por ejemplo, la UOM estaba suscripta y que compraban más de 50 ejemplares, que distribuían en las seccionales de todo el país. A su vez, el periodista Santiago Senén González destaca que los mismos eran utilizados por distintos dirigentes gremiales y periodistas, fuerzas de seguridad y empresas. ${ }^{11}$

En una notificación de 1972 dirigida a los suscriptores, se menciona a las personas e instituciones adheridas. Allí se señalan diversos sindicatos y federaciones obreras, como La Unión Ferroviaria, Petroleros del Estado, el sindicato de Luz y Fuerza de Córdoba, diarios como La Opinión o Clarín, hasta empresas multinacionales como FIAT SOMECA, The Bank of Tokio LT, la Embajada de la República Federal Alemana, y servicios de inteligencia como la S. I. D. E. y la S. I. E. ${ }^{12}$

21 Los Informes Laborales tuvieron dos etapas, la primera abarcó desde su nacimiento en febrero de 1960 hasta su cierre en $1976 .{ }^{13}$ La segunda etapa comprendió los años 1982 hasta 1989. A partir de 1987, al morir Leonardo Dimase, su hijo Miguel se hizo cargo de los DIL hasta que finalmente se ve obligado a cerrarlo debido a la crisis económica por la que atravesaba en ese momento el país.

\section{Lugares de consulta de los DIL}

22 La colección completa de los Informes Laborales, tanto de la primera como de la segunda época, puede encontrarse en el Centro de Estudios e Investigaciones Laborales del Consejo Nacional de Investigaciones Científicas y Técnicas (CEIL-CONICET), subsuelo de Saavedra 15, Ciudad Autónoma de Buenos Aires, y en la Biblioteca de la Confederación General del Trabajo de la República Argentina (CGT), Azopardo 802, 3er piso.

Colecciones no completas de los Informes Laborales, así como otras publicaciones no periódicas de DIL (Convenios, Remuneraciones, Guía de Organizaciones Gremiales), pueden encontrarse en los Archivos de la Universidad Di Tella, en el Centro de Documentación e Investigación de la Cultura de Izquierdas en la Argentina (CeDInCI), en la Biblioteca del Ministerio de Trabajo y en la Biblioteca Nacional Mariano Moreno.

\section{Leonardo Dimase}

Leonardo Enrique Dimase nació en Pergamino el 4 de julio de 1920 y murió el 9 de mayo de 1987. Fue profesor titular de Derecho del Trabajo en la Facultad de Derecho de la Universidad de Buenos Aires (UBA) y de la Universidad Argentina de la Empresa (UADE). Le faltaron pocas materias para recibirse de abogado pero, según relata Miguel, tuvo que abandonar sus estudios para trabajar. 
gremial argentino. Producto de este encuentro -realizado en junio de 1964 por el Centro Argentino por la Libertad de la Cultura, filial argentina del Congreso por la Libertad de la Cultura, de París- se editó el libro La situación gremial argentina. Años después, Dimase fue convocado junto a otros periodistas, sindicalistas, empresarios, funcionarios y políticos para reflexionar acerca de la participación de los trabajadores en la gestión económica. En esa oportunidad señaló:

"Históricamente está demostrado que, en el sistema capitalista, condiciones de elevado desarrollo suficientemente asentado -con su consecuente relativa satisfacción social- son las únicas que permiten la institucionalización de algunas formas de cogestión. En tales casos el trabajador se convierte en una especie de "socio muy menor" del empresario al par que éste obtiene un importante "reaseguro" contra futuras reivindicaciones de aquél. Fundamentalmente, los mecanismos de "participación" en los países donde se dan las condiciones aludidas, logran la "integración" del trabajador al sistema capitalista, con sus naturales consecuencias. La experiencia también indica que en aquellos lugares en que no se ha alcanzado un alto grado de desarrollo, todo intento de cogestión implica una mistificación cuya única víctima es el trabajador." (Selser, 1970, pp. 132-133)

Durante el gobierno de Arturo Illia volvió a la función pública. Trabajó en el Ministerio de Asistencia Social y Salud Pública de la Nación, a cargo de Arturo Oñativa. Después del golpe de Estado de 1966, Dimase presentó su renuncia pero no se la aceptaron, motivo por el que siguió trabajando allí hasta después de la muerte de Juan D. Perón en el año 1975. Luego se produjo el cierre del DIL. Durante este período iba recibiendo encargos o escribiendo notas internacionales. Con el advenimiento de la democracia, fue representante permanente del gobierno argentino ante la OIT durante el gobierno de Raúl Alfonsín. Rechazó varias veces el ofrecimiento de estar a cargo del Ministerio de Trabajo. Finalmente, Leonardo Dimase murió en el año 1987. 


\section{Conclusiones}

30 para el estudio del movimiento obrero argentino, entre 1960 y 1989. La posibilidad de encontrar en los Informes Laborales información detallada sobre conflictos, congresos, elecciones, asambleas, cantidad de afiliados, convenios, leyes, decretos, entre otros, configuran a estos documentos como una fuente relevante.

31 No nos es ajeno que, como cualquier tipo de documentos, los Informes Laborales deben ser cruzados con otros como los citados en la introducción: diarios, revistas, publicaciones oficiales, archivos desclasificados de las fuerzas de seguridad, entre otros. Asimismo, reconstruimos el origen de estos documentos, que nos permite comprender quién los hacía y a quiénes iba dirigido, especialmente la intención de su fundador de servir de conexión entre el mundo obrero y el mundo empresarial. Finalmente pudimos dar a conocer los lugares actuales de consulta de estos documentos.

\section{BIBLIOGRAFÍA}

Izaguirre, I.; Aristizábal, Z. (2002). Las luchas obreras 1973-1976. Los alineamientos de la clase obrera durante el gobierno peronista. Nuevas consideraciones teórico-metodológicas para el estudio de los conflictos obreros. Documentos de Trabajo № 17, [en línea], Instituto de Investigaciones Gino Germani, disponible en <http://lanic.utexas.edu/project/laoap/iigg/ dt17.pdf> [consulta: 10 de enero 2012]

Dawyd, D. (2011). Sindicatos y política en la Argentina del Cordobazo. El peronismo entre la CGT de los Argentinos y la reorganización sindical (1968-1970). Buenos Aires: Editorial Pueblo Heredero.

Duval, N. (1974). Argentina: sindicatos y movimientos de masas. En A. Pla, Historia del Movimiento Obrero. Tomo V, № 95, pp. 289-320). Buenos Aires: CEAL.

Gallego, M. (2008). Clase obrera, dictadura y resistencia (1976-1983). En L. Pasquali (Comp.), Historia social e historia oral. Experiencias en la historia reciente de Argentina y América Latina. Rosario: Homo Sapiens.

McGuire, J. W. (1992). The causes of strikes in Argentina, 1984-1991. Ponencia presentada en el XVII International Congress of the Latin American Studies Association, Los Angeles, California.

Nassif, S. (2012). Tucumanazos. Una huella histórica de luchas populares 1969-1972. Tucumán: Universidad Nacional de Tucumán, Facultad de Filosofía y Letras.

Selser, J. (1970). Participación de los trabajadores en la gestión económica. Buenos Aires: Ediciones Libera.

\section{Fuentes orales}

- Entrevista realizada a Santiago Senén González, periodista y estudioso del movimiento obrero argentino, realizada por los autores, Buenos Aires, 11 de septiembre de 2013. 
- Entrevista realizada a Miguel Dimase, hijo de Leonardo Dimase, parte del equipo de trabajo de los DIL, Buenos Aires, 28 de septiembre de 2013.

\section{Documentos}

Documento Dimase, Leonardo, Documentación e Información Laboral, julio de 1972.

Informe Nucleamientos Sindicales, de 1972.

La Opinión, martes 15 de agosto de 1972, p. 10.

DIL, № 199-200, septiembre-octubre de 1976.

Documentación e Información Laboral, Informe Laboral, №1, 10 de marzo de 1960.

\section{NOTAS}

1. Entre las excepciones pueden citarse ciertos documentos conservados en el Centro de Documentación e Investigación de la Cultura de Izquierdas en la Argentina (CeDInCI), en los archivos de la Universidad Di Tella y en la Biblioteca del Ministerio de Trabajo. En la actualidad el archivo de la CGT de Azopardo se encuentra en proceso de reordenamiento. Se está avanzando en la reclasificación y en la digitalización de su acervo documental, por lo que esperamos que en un futuro se pueda consultar.

2. Siguiendo a Izaguirre y Aristizabal (2002, p. 20-21), el periódico "para casi la totalidad de los problemas que se plantean los científicos sociales, no sólo es casi el único registro del orden de lo real que se ofrece en forma cotidiana, con la ventaja adicional de ser archivable y de acceso relativamente sencillo [...] la prensa escrita ha logrado constituirse en un medio estandarizado de una gran masa de información de todo tipo. [...] Los riesgos acerca de su carácter "intencional" o "ideológico" no son mayores que los de otras fuentes y pueden ser disminuidos manteniendo con ese material una relación crítica, y construyendo un instrumento de captación con criterios rigurosos, puesto que, salvo en situaciones de realidad excepcionales [...] el sesgo ideológico de una publicación se mantiene a lo largo del tiempo, y opera sobre la totalidad de la información en una sección similar". (Resaltado en el original).

3. Como el "Archivo policial de la DIPBA (ex Dirección de Inteligencia de la Policía de la Provincia de Buenos Aires) que se encuentra en la sede de la Comisión Provincial por la Memoria [que] contiene legajos por empresa, también legajos por gremios $u$ organizaciones (UOM, SMATA, AOT, 62 Organizaciones) y distintos tipos de dossier: panorama gremial, desocupación, actividad industrial, huelgas y conflictos" (Gallego, 2008, p. 121). Cabe aclarar que puede encontrarse información no solo de Buenos Aires, sino también de sindicatos, organizaciones y dirigentes de otras provincias.

4. Entre estos años, más precisamente entre 1966 y 1967, se editó la revista Estudios Sindicales, dirigida por Roberto Cappagli, objeto de un trabajo de investigación en elaboración.

5. Según Miguel Dimase (hijo de Leonardo Dimase), en febrero de 1960 salió el número 0, del que no conserva ningún ejemplar. Se habrían hecho entre 1000 y 2000 ejemplares. Asimismo, señala que tuvieron un problema con la impresión de la revista por lo que su padre junto a su familia tuvieron que arreglarlos uno por uno en su casa (entrevista realizada a Miguel Dimase, septiembre de 2013).

6. Documentación e Información Laboral, Informe Laboral, № 1, 10 de marzo de 1960, p. 2.

7. Documento Dimase, Leonardo, Documentación e Información Laboral, julio de 1972.

8. Cabe aclarar que el DIL tuvo diversas oficinas, sujetas a la coyuntura económica.

9. Entrevista realizada a Miguel Dimase, septiembre de 2013. 
10. Documento Dimase, Leonardo, Documentación e Información Laboral, julio de 1972. Asimismo, sobre el Informe Nucleamientos Sindicales, de 1972, el diario La Opinión publicó una reseña de más de media página: "Una breve historia de la evolución de los núcleos políticos que integran el movimiento obrero argentino, apoyados en una serie de cuadros estadísticos, ha sido publicada por una organización periodística especializada en las informaciones vinculadas al mundo del trabajo, Documentación e Información Laboral (DIL). El documento se integra con un rápido racconto de la conformación de los nucleamientos sindicales durante los últimos 15 años, efectuado con un criterio objetivo. El desarrollo del proceso tiene un alto interés para evaluar la situación actual del movimiento obrero y las raíces de cada sector presente en el pasado inmediato de esas luchas, esencialmente políticas [...] Las publicaciones DIL inauguraron con este Documento un nuevo tipo de servicio, que se añade a los que ofrecen habitualmente, relacionados con convenios, asambleas y conflictos sindicales, y con interpretaciones periódicas de las vinculaciones entre la situación del movimiento obrero y el panorama político" (La Opinión, martes 15 de agosto de 1972, p. 10).

11. Entrevista realizada a Miguel Dimase, septiembre de 2013 y entrevista realizada a Santiago Senén González, septiembre de 2013.

12. DIL, Documentación de Información Laboral, Buenos Aires, julio de 1972.

13. Las palabras de Dimase, en el último informe laboral, fueron: "La conjunción de diversas circunstancias (situación económico-financiera; suspensión de la actividad gremial y política; entre otros) nos obliga a plantearnos la necesidad de un alto en nuestro desenvolvimiento. En efecto; con las entregas del presente mes dejarán de aparecer, transitoriamente, las distintas publicaciones mensuales de DIL [...] Hacemos propicia la oportunidad para reiterarles el agradecimiento por las constantes muestras de adhesión para la tarea que, desde hace más de 16 años, viene desarrollando DIL en nuestro país. Cordialmente, La Dirección, Buenos Aires, 31 de octubre de 1976" (DIL, № 199-200, septiembre-octubre de 1976).

14. Entrevista realizada a Miguel Dimase, septiembre de 2013 y entrevista realizada a Santiago Senén González, septiembre de 2013.

\section{RESÚMENES}

En el presente artículo nos proponemos dar cuenta de la importancia del Servicio de Documentación e Información Laboral (DIL), dirigido por Leonardo Enrique Dimase, como posible fuente para el estudio del movimiento obrero argentino, en dos períodos: 1960-1976 y 1982-1989. Con tal motivo, reconstruimos el origen de estos documentos, con vistas a comprender quién los hacía y a quiénes iba dirigido. También damos cuenta de los lugares actuales de consulta de estos documentos. Para ello nos valemos de los pocos trabajos que mencionan a esta fuente, entrevistas a quienes conocieron a su director y una revisión exhaustiva de los documentos producidos en los años mencionados.

This paper expects to account for the importance of DIL (Servicio de Documentación e información Laboral, Job Documentation and Information Service) led by Leonardo Enrique Dimase, as possible source for the study of the working class movement in Argentina, in two periods: 1960-1976 and 1982-1989. For this purpose we reconstruct the origin of these documents in order to understand who made them and to whom they were addressed. We also account for current places where these documents can be consulted. To do this we make use of the few works that mention this 
source, interviews with those who knew the director and a thorough review of the documents produced between such years.

\section{ÍNDICE}

Keywords: working class movement, labor, documents, archives

Palabras claves: movimiento obrero, trabajo, documentos, archivos

\section{AUTORES}

\section{DARÍO DAWYD}

Universidad Nacional de La Matanza (UNLaM). Becario postdoctoral del CONICET (Consejo Nacional de Investigaciones Científicas y Técnicas) en el Centro de Estudios e Investigaciones Laborales (CEIL), Argentina.

Correo electrónico: dawydario@gmail.com

\section{SILVIA NASSIF}

Becaria del CONICET (Consejo Nacional de Investigaciones Científicas y Técnicas)- Instituto de Investigaciones Históricas “Dr. Ramón Leoni Pinto” Universidad Nacional de Tucumán, Argentina.

Correo electrónico: nassifsilvia@gmail.com 